\title{
Research on Application of Digital Platform Based on Community Network Marketing
}

\author{
Zhou Li \\ Jinan Vocational College, Shandong, Jinan250000 \\ hunter2011@foxmail.com
}

Keywords: Digital community, Digital platform, Network marketing.

\begin{abstract}
The digital platform based on community network marketing is a special network marketing platform. It is the business platform which uses the community or residence community as service unit, depends on MAN or private network of bandwidth, and as integrating consumption as operation concept to meet the consumer demand of community resident. The paper proposes new digital platform based on community network marketing, and designs and implements it in community marketing digital platform program of Shanghai Eastern Digital Community Corporation, which has great academic significance and actual application value.
\end{abstract}

\section{Introduction}

Network marketing appears with the development of information technology. The application and development of computer technique, network, communication and multi-media technology composes technical basis of network marketing. Various services of INTERNET reflects the functions of connecting, transmitting, interacting and storing various information, which makes INTERNET have the ability of commercial transaction and interactive communication.

The digital platform based on community network marketing can meet the requirement of convenient, easy and comfortable life which is expected by community residents. By the establishment and promotion of the platform, the community resident can meet the daily service requirements by keeping indoors. The consumption group of the new network marketing platform is the residents in the community, which is more clear compared with traditional network marketing platform. The participation of property management increases the trust of users for interaction of community network marketing. It uses providing service relating to community residents as profit point. The operation direction is clear and the operation benefit is evident. Digital platform based on community network marketing has significant advantage for safety, credibility and delivery system, which can develop rapidly. At present, many digital platforms based on community network marketing face some problems, which is the reason of influencing the development of platform. So the paper researches the digital platform based on community network marketing, for finding the factors influencing the development of digital platform based on community network marketing, and proposing the solution.

\section{Development Status of Digital Platform Based on Community Network Marketing}

With the appearance of intelligent residence community, the digital platform based on community network marketing develops new service filed---community e-commerce. In community e-commerce, Beijing is advanced, and the idea of developing community service information network of the capital was proposed in 1995. With many years of efforts, experiment pilots are established such as Electronic Comprehensive Calling Network Center of Dashilar Community in Xuanwu District, Red-yellow-blue Network center in Xicheng District, Tsinghua Park Community Service Center in Haidian District and Liangxiang Xiluyuan Information Community in Fangshan District. The experiment pilots accumulate experience, and are the rudiment of community network marketing in China, and it lays good foundation for starting 
community service information engineer. In actual application, the exploratory model has appeared in some cities in China such as Community Public Service Platform in Beijing_ 96156 platform, and Community Information Service Center in Chengdu.

The consumption group of the new community network marketing platform is the direct residents in the community, which is more clear than the traditional network marketing platform. The participation of property management increases the trust of users for interaction of community network marketing. It uses providing service relating to community residents as profit point. The operation direction is clear and the operation benefit is evident.

\section{Design Idea and Application of New Digital Platform}

In order to solve the problems of digital platform based on community network marketing, the paper suggests establishing new digital platform. And the idea is to depend on the scale community websites, establish e-commerce information virtual system platform and payment system facing PC, mobile phone and TV. The design idea and application of the platform is shown in Figure 1 and Figure 2.

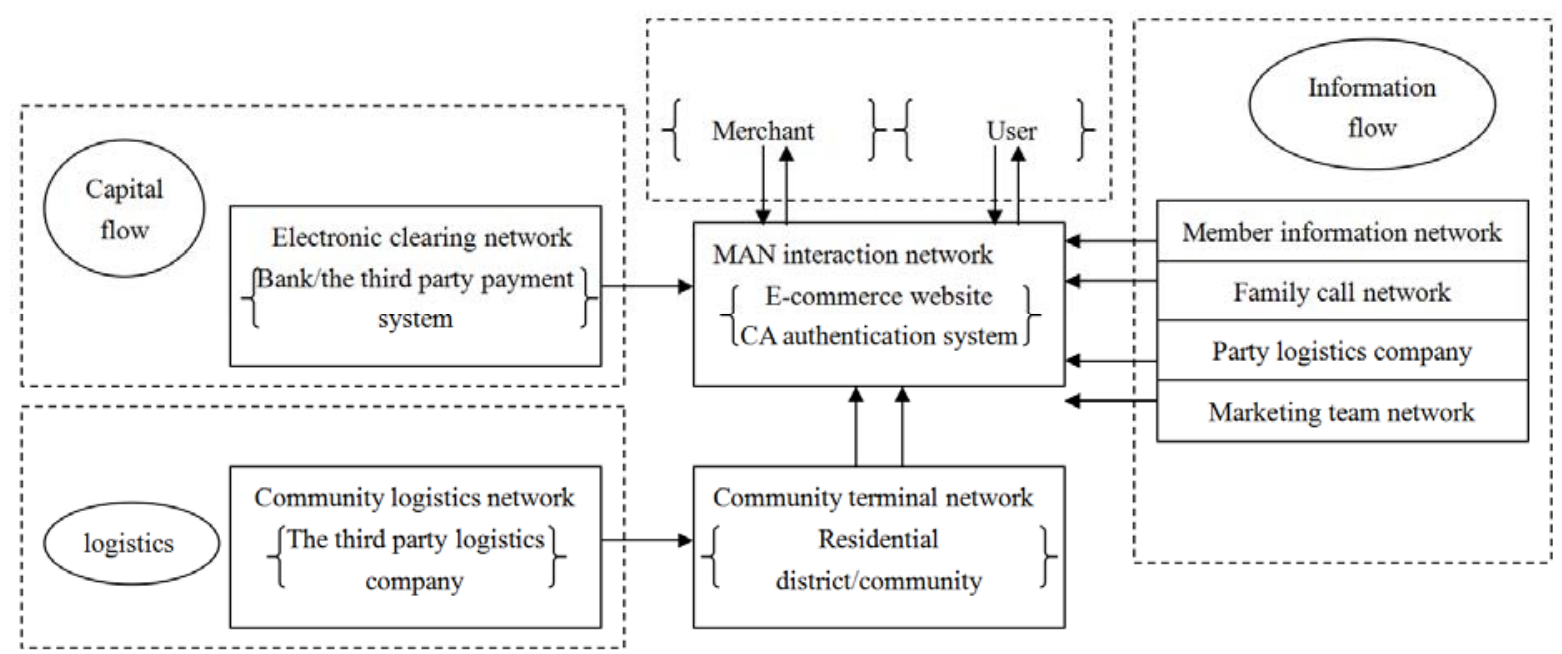

Figure 1 Design idea of new digital platform

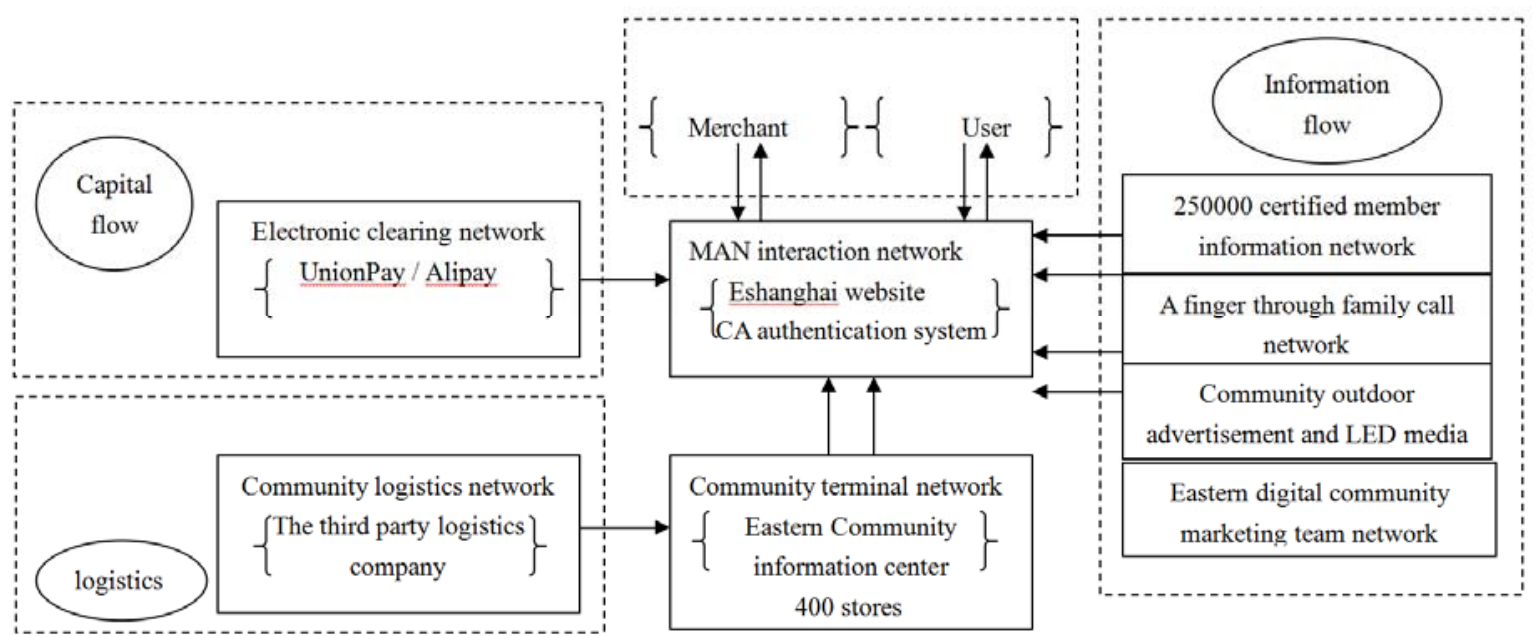

Fig. 2 Design idea of digital platform in Shanghai eastern digital community company

\section{System Architecture of Platform}

The objective of establishing platform and actual requirement of user group should be based on rational model, and the overall architecture of platform system should be designed properly. The 
overall architecture is a functional structure figure, which includes the following levels and modules.

Common basis platform. It provides the basic functions of the system including door, work flow, message bus and DNS/DHCP.

Operation support system. It provides basic support service of operation including business, customer service and delivery.

Business support system. It completes basic supports of business operation including statistics, finance and settlement.

Management support system. It completes the business of basic management support including finance and logistics.

User service layer. It provides various services facing users. Under the support of other support systems, the services can be expanded and adjusted.

External system. It includes other systems which need to use relative information for interaction in service process.

\section{Network Topology}

The data center of the platform is placed in Shanghai Telecom IDC Room. And VIP room is rent to place various servers required by the platform. And it enjoys $100 \mathrm{M}$ internet exit bandwidth alone. The project management center uses MPLS VP technique 10M to connect with IDC room by optical fiber. And the operations including management, content update and analysis are implemented to master the condition of platform.

\section{Key Function Components of Platform}

The establishment content of platform system is to establish new digital platform support system including basic software and hardware system construction in Central engine room, and software configuration and second development of support platform.

Basic software and hardware system construction. The network system includes two Routers, core exchange boards and firewalls. The host system includes 3 database servers and 18 various application servers and function servers. The storage system includes SAN memory system, and the total memory capacity is 3TB. The total memory capacity of tape library is 3TB. Basic software includes operation system software, database software, development tool software and business e-commerce software.

Second development of software includes infrastructure platform, operation support system, business support system, management support system, user service level and external system.

For customer analysis, according to the design requirements of integrating community terminal network, community logistics network, metropolitan interaction network, family calling network, electronic settlement network, member information network, community media network and marketing team network, and integrating information flow, capital flow and logistics flow, the key function components of platform are added to support the above model innovation.

\section{Conclusions}

Digital platform based on community network marketing is a special community network marketing platform. It is established based on the online shopping requirement of community residents. And it is improved network marketing model. In operation process, it solves the closed loop problem, non-popular problem and imperfect service system in actual operation of the platform. It provides a new marketing channel for small and medium enterprises on the platform under the model. And the accumulated member information contributes to information mining and requirement refinement for middle and small-sized enterprises. 


\section{Reference}

[1] Center for Research in Electronic Commerce of the University of Texas at Austin. Research Priorities in Electronic Commerce. Workshop Report(Sponsored by the National Science Foundation), 1999,1

[2] Clinton Wilder, Bruce Caldwell.More Than Electronic Commerce Information-Week.1997,12

[3] Ravi Kalakota. Electronic Commerce-Amanager's Guide.Addison-Wesley Longman,Inc.,1997

[4] E. Turban et al..Electronic Commerce: A Managerial Perspective. Prentice Hall Press, 2006

[5] Lee M KO, Turban E, A Trust Model for Consumer Internet Shopping. International Journal of Electronic Commerce, 2001;6(1)

[6] P Resnick, R Zeck hauser, E Frienman, K Kuwabara. Reputation System. Communications of the ACM, 2000, 43(12) 